\title{
Conformal embeddings and quantum graphs with self-fusion
}

\section{R. Coquereaux ${ }^{1}$}

Centre de Physique Théorique(CPT), Luminy, Marseille

\begin{abstract}
After a short description of the notion of quantum subgroups and quantum modules of quantum groups at roots of unity, in the framework of category theory, and a presentation of the known classifications (that we relate with the theory of conformal embeddings), we sketch several general methods of study and illustrate them on the particular example of a quantum subgroup of the non simple Lie group $S U(2) \times S U(3)$ stemming from a conformal embedding of the later, at level $(16,6)$, into $E_{8}$. The graph describing its module structure over the corresponding fusion algebra incorporates several known components graphs of type $S U(2)$ and $S U(3)$, and it has self-fusion, but some of its components don't.

Keywords: quantum symmetries; modular invariance; conformal field theories; quantum groupoïds.
\end{abstract}

\section{General presentation}

To any conformal embedding $K \subset G$, interpreted either in terms of affine Lie algebras or in terms of quantum groups at roots of unity, one can associate a module-category $\mathcal{E}(K)$ endowed with an action of a modular category $\mathcal{A}(K)$ defined either as the fusion category of $K$ at some level, or as a category of representations with non-zero $q$-dimension of the quantum group $K$, at a root of unity determined by the level of the embedding. These module-categories enjoy self-fusion: such "quantum modules" can therefore be considered as "quantum subgroups" of the group $K$. After a brief description of the general situation and a summary of the known classification of quantum modules when $K$ is $S U(2), S U(3)$ or $S U(4)$, classification that associates a graph describing the action of the generators of the Grothendieck ring of $\mathcal{A}(K)$ on the simple objects of $\mathcal{E}(K)$, we give a classification of quantum subgroups, stemming from the known

\footnotetext{
${ }^{1}$ Lecture presented at the XVII Coloquio Latinoamericano de Algebra, Medellin, Colombia, July 23 to $27,2007$.
} 
conformal embeddings. Then, we study one particular example where the given classical Lie group $K$ is not simple, namely $K=S U(2) \times S U(3)$, and where $G=E_{8}$. It gives rise to a quantum subgroup of $K$ whose graph components incorporate the Dynkin diagrams $E_{7}=\mathcal{E}_{16}(S U(2))$ and $D_{10}=\mathcal{D}_{16}(S U(2))$, but also several known graphs of type $S U(3)$, namely $\mathcal{D}_{6}(S U(3))$ and $\mathcal{D}_{6}{ }^{c}(S U(3))$. It is known that $D_{10}$ and $\mathcal{D}_{6}(S U(3))$ enjoy self-fusion, but $E_{7}$ and $\mathcal{D}_{6}{ }^{c}(S U(3))$ don't. This suggests that conformal embeddings not only give rise to quantum subgroups of Lie groups $K$ (which are not necessarily simple) but, when followed by contraction or twisting, they may provide a way to obtain all quantum modules.

1.1. Categorical description of the framework. Category theory offers a synthetic presentation of the framework and we present it here in a few lines. However most of the constructions that will be found in this paper are made at the level of groups and rings, so that familiarity with category theory itself is not required to read this paper.

The starting point is the fusion category $\mathcal{A}_{k}=\mathcal{A}_{k}(K)$ associated with a Lie group $K$ and an integer $k$ called the level (see for instance [28]). This category, both monoidal and ribbon, can be defined either ${ }^{1}$ in terms of representation theory of an affine Lie algebra (simple objects are highest weight integrable irreducible representations) or in terms of representation theory of a quantum group at a root of unity $q$ determined by the level (simple objects are irreducible representations of non-vanishing quantum dimension). One should keep in mind the distinction between this category (with its objects and morphisms), its Grothendieck ring (the fusion ring), and the Cayley graph describing multiplication by its generators, but they are denoted by the same symbol. In simple terms, and at the level of rings, simple objects of this category are irreducible representations of a Lie group $K$ that are such that their level is bounded by a given integer $k$ (in the case of groups of type $S U(n)$, the level of an irreducible representation specified by a Young diagram is given by its maximum size, the width or the height of the diagram).

The next ingredient is an additive category $\mathcal{E}_{k}=\mathcal{E}_{k}(K)$, on which the previous monoidal category acts. Action of a monoidal category on a category is defined whenever we are given a (monoidal) functor from $\mathcal{A}_{k}$ to the (monoidal) category of endofunctors of $\mathcal{E}_{k}$. The reader can think of this situation as being an analogue of the action of a group on a given space. It may be sometimes interesting to think that $\mathcal{E}$ can be acted upon in more than one way, so that we can think of the action of $\mathcal{A}_{k}$ as a particular enrichment of $\mathcal{E}_{k}$, or that $\mathcal{E}_{k}$ is an actegory (a nice substantive coined by R. Street). In general $\mathcal{E}_{k}$ has no-self-fusion (no compatible monoidal

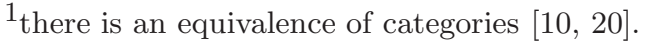


structure) but in some cases it does. Here again, the category itself, its Grothendieck group, and the graph describing the action of generators of $\mathcal{A}_{k}(K)$ are denoted by the same symbol. The later graph will be called the McKay graph of $\mathcal{E}_{k}$, or simply, "the quantum graph". Using Ocneanu terminology [23] [24], one say that such an $\mathcal{E}_{k}$ is a quantum module of $K$ (at some root of unity), and if it happens that this quantum module has self-fusion, it is called a quantum subgroup of $K$. In the classical situation, this indeed happens when $\mathcal{E}$ is a subgroup: Take for instance $K=S U(2)$, $k=\infty, \mathcal{A}$ the ring generated by all irreducible representations of $S U(2)$ (they are specified by an integer or half-integer $s$, the spin), and $\mathcal{E}$ the ring generated by irreducible representations (up to equivalence) of some binary polyhedral subgroup.

The last ingredient is the centralizer (or dual) category $\mathcal{O}=\mathcal{O}\left(\mathcal{E}_{k}\right)$ of $\mathcal{E}_{k}(K)$ with respect to the action of $\mathcal{A}_{k}(K)$. It is monoidal and comes with its own ring, called the Ocneanu algebra of quantum symmetries, which is a bimodule over the fusion ring, and a graph (the Ocneanu graph) which is the Cayley graph describing multiplication by the generators. See [22, 2, 25].

The category $\mathcal{A}_{k}$ can be realized as the tensor category of representations of a certain weak Hopf algebra, this is a theorem proved by Y. Hayashi [16]. One way to obtain a realization of the above collection of data is therefore to construct a finite dimensional weak bialgebra $\mathcal{B}$, which should be such that $\mathcal{A}_{k}$ can be realized as $\operatorname{Rep}(\mathcal{B})$, and also such that $\mathcal{O}$ can be realized as $\operatorname{Rep}(\widehat{\mathcal{B}})$, where $\widehat{\mathcal{B}}$ is the dual of $\mathcal{B}$. These two algebras are finite dimensional, actually semisimple in our case, and one algebra structure (say $\widehat{\mathcal{B}}$ ) can be traded against a coalgebra structure on its dual. $\mathcal{B}$ is a weak bialgebra, not a bialgebra, because $\Delta \mathbb{1} \neq \mathbb{1} \otimes \mathbb{1}$, where $\Delta$ is the coproduct in $\mathcal{B}$, and $\mathbb{1}$ is its unit. $\mathcal{B}$ is not only a weak bialgebra but a weak Hopf algebra (a quantum groupoid): one can define an antipode, with the expected properties. Such quantum groupoids $\mathcal{B}\left(\mathcal{E}_{k}(K)\right)$ defined as module-categories over a given fusion algebra $\mathcal{A}_{k}(K)$ attached to a Lie group $K$ and a positive integer $k$ are sometimes called "Ocneanu quantum groupoids of type $K$ ". A description of this setup for the case of $S U(2)$, in the framework of category theory, was presented by $[25,11]$.

Let us conclude this presentation by mentioning that simple objects of $\mathcal{E}_{k}$ can be thought as would-be spaces of sections for quantum bundles over the quantum space determined by the pair $\left(\mathcal{A}_{k}, \mathcal{E}_{k}\right)$, and therefore as modules over an algebra $\mathcal{F}$, actually a Frobenius monoid, in the monoidal category $\mathcal{A}_{k}$. We shall not use Frobenius algebras in our article but we should nevertheless mention that such a point of view was developped in [12], [25] and that [21] proved that $\mathcal{E}_{k}$ is monoidal (existence of self-fusion, so that it is a "quantum subgroup", not only a "quantum module") if and only if $\mathcal{F}$ is commutative. Terminological warning: in the older paper [21] 
this commutativity property was part of the definition and such algebras were called rigid, both requirement and terminology were later abandoned.

1.2. The classification problem. The problem is to obtain a description of all possible quantum modules $\mathcal{E}(K)$, where $K$ is some arbitrarily given semi-simple (or reductive) Lie group. For each of them, ideally, one wants to be able of giving a set of generators for the Grothendieck group of the category, a collection of matrices implementing the various algebra and module structures, and a presentation of the associated quantum groupoid (multiplication on $\mathcal{B}$ and on its dual, pairing).

In order to describe these related structures, one introduces the following notations and terminology: fusion matrices $N_{n}=\left(N_{n p q}\right)$ encode the structure constants of the ring $\mathcal{A}_{k}$, annular matrices $F_{n}=\left(F_{n a b}\right)$ encode the structure constants of the module $\mathcal{E}_{k}$ over the ring $\mathcal{A}_{k}$, graph matrices $G_{a}=\left(G_{a b c}\right)$ describe the monoidal structure of $\mathcal{E}_{k}$ when it exists (existence of self-fusion), matrices of quantum symmetries $O_{x}=\left(O_{x y z}\right)$ encode the structure constants of the ring $\mathcal{O}$, toric matrices $W_{x y}=\left(W_{x y}\right)_{m n}$ and double fusion matrices $V_{m n}=\left(V_{m n}\right)_{x y}$ related to one another by $\left(V_{m n}\right)_{x y}=\left(W_{x y}\right)_{m n}$ describe the bi-module structure of $\mathcal{O}$ over $\mathcal{A}_{k}$, dual annular matrices $S_{x}=\left(S_{x}\right)_{a b}$ describe the module structure of $\mathcal{E}_{k}$ over $\mathcal{O}$, Ocneanu cells $\{a, n, b ; c, x, d\}$ give the pairing between the bialgebra $\mathcal{B}$ and its dual [23]. In this article, indices $m, n, p \ldots$ label simple objects of $\mathcal{A}_{k}$, $a, b, c \ldots$ simple objects of $\mathcal{E}_{k}$ and $x, y, z, \ldots$ simple objects of $\mathcal{O}$.

\section{Quantum modules and quantum subgroups}

\subsection{A brief description of the $S U(2), S U(3), S U(4)$ cases.}

$S U(2)$. In this case, the classification of quantum modules is equivalent to the classification of modular invariant partition functions for WZW conformal field theories of type $S U(2)$ which was obtained long ago by theoretical physicists: this is the ADE classification found by [3].

The module structure of $\mathcal{E}_{k}$ is fully described, in this case, by the annular matrix $F_{1}$, since the character ring of $S U(2)$ has only one generator. From quantum group theory we know that the quantum dimension of the fundamental representation is also the Perron-Frobenius norm $\beta$ of $F_{1}$ and it is smaller than 2 when $q$ is a root of unity. Moreover, from the fact that matrix elements of $F_{1}$ are non-negative integers, one is immediately led to a classification in terms of Dynkin diagrams (set $F_{1}=2$ Cartan matrix $-\mathbb{1}$ ). 
The rigidity condition ${ }^{2}$ in the case of $s l(2)$ implies that the annular matrix $F_{1}$ is symmetric, this condition excludes the non simply laced diagrams $B_{r}, C_{r}, F_{4}$ and $G_{2}$. We are left with the $A D E$ diagrams and the tadpoles. A detailed analysis of the situation ([22], [25]) shows that tadpole graphs do not give rise to any category endowed with an action of $\mathcal{A}_{k}(S U(2))$. In those $S U(2)$ cases, the McKay graphs of the quantum modules $\mathcal{E}_{k}$ are precisely given by the simply laced Dynkin diagrams. Among them, $E_{7}$ ( level is 16) and $D_{2 s+1}$ (level is $k=4 s-2$ ), do not have self-fusion, but the others cases do: $A_{r}$ (level is $k=r-1$ ), $D_{2 s}$ (then $\left.k=4 s-4\right), E_{6}$ (then $k=10$ ), and $E_{8}$ (then $k=28$ ) indeed describe $S U(2)$ quantum subgroups. At the level of graphs, the $D$ diagrams (even or odd) are $\mathbb{Z}_{2}$ orbifolds of the $A$ diagrams at the same level. All these quantum modules of $S U(2)$, arising when $q^{2+k}=-1$, can be obtained as follows.

The $\mathcal{A}_{k}(S U(2))=A_{k+1}$ are immediately obtained by truncation of the Weyl chamber of $S U(2)$ at level $k$. $\mathcal{E}_{k=10}(S U(2))=E_{6}$ which is a module over $\mathcal{A}_{10}(S U(2))=A_{11}$ is a quantum subgroup obtained from the conformal embedding of $S U(2)_{10}$ in $\operatorname{Spin}(5) \simeq S p(2) . \mathcal{E}_{k=28}(S U(2))=E_{8}$ which is a module over $\mathcal{A}_{28}(S U(2))=A_{29}$ is a quantum subgroup obtained from the conformal embedding of $S U(2)_{10}$ in $G_{2}$. $\mathcal{D}_{k=4}(S U(2))=D_{4}$ which is a module over $\mathcal{A}_{4}(S U(2))=A_{5}$ is a quantum subgroup obtained from the conformal embedding of $S U(2)_{4}$ in $S U(3)$, this is the smallest member of the $D_{\text {even }}$ series, which has self-fusion; the other members of this series are not obtained from a (direct) conformal embedding. Indeed, conformal embeddings of $S U(2)_{k} \times S U(k)_{2}$ in $S U(2 k)$ give rise to quantum subgroups of the semi-simple (but not simple) quantum group $S U(2) \times S U(k)$ and the property of self-fusion for it is automatic, but it may be lost in the process of contraction with respect to either of the simple factors : take $k$ even (if it is odd one does not obtain any new module), then if we contract with respect to $S U(k)$, we obtain quantum modules of $S U(2)$ and their McKay diagrams are Dynkin diagrams of type $D_{s}$, with $s=k / 2+2$, and they have self-fusion if $s$ is even but they don't when $s$ is odd. Finally we have the conformal embedding of $S U(2)_{16} \times S U(3)_{6}$ into $E_{8}$. We shall study this example in the last section and see that it gives rise to a quantum subgroup of $S U(2) \times S U(3)$ whose McKay graph contains one graph $D_{10}=$ $\mathcal{D}_{16}(S U(2))$, two graphs $E_{7}=\mathcal{E}_{16}(S U(2))$, one graph $\mathcal{D}_{6}(S U(3))$ together with its module $\mathcal{D}_{6}{ }^{c}(S U(3))$. Contraction with respect to $S U(3)$ gives one $D_{10}$ (which has self-fusion) and two $E_{7}$ (which has not). Contraction with

\footnotetext{
${ }^{2}$ Rigidity of the category $\mathcal{A}_{k}$ (existence of duals) implies that fusion ring is "rigid" i.e., that $\left(N_{\bar{n}}\right)_{p q}=\left(N_{n}\right)_{q p}$, where $\bar{n}$ refers to the dual object (conjugate representation), or that it is based $\mathbb{Z}_{+}$ring in the sense of [25]. At the level of modules, this property also implies that the module $\mathcal{E}$ is rigid, in other words: $\left(F_{\bar{n}}\right)_{a b}=\left(F_{n}\right)_{b a}$.
} 
respect to $S U(2)$ gives also two graphs of $S U(3)$, one with self-fusion $\left(\mathcal{D}_{6}\right)$ and one without $\left(\mathcal{D}_{6}{ }^{c}\right)$.

Starting from a non-simple conformal embedding i.e., $K \subset G$ with $G$ simple, but $K$ semi-simple, non simple, one can perform a contraction at the level of modular invariants (the above example, after subtraction of the $D_{10}$ part provides a standard way to obtain the modular invariant of $E_{7}$, as described, for instance, in [8]) but if we do not contract, we obtain a quantum subgroup of the semi-simple group $K$.

$S U(3)$. In this case, the classification of $\mathbb{Z}_{+}$modules over the corresponding fusion rings at level $k$ is not tractable, and would not be useful, anyway, for our purposes. However there is another route stemming from the classification (mostly based on arithmetical considerations) of modular invariant partition functions of type $S U(3)$, obtained by T. Gannon [13]. Using a variety of techniques, one then obtains the McKay graphs for the quantum modules of type $S U(3)$ : they are given by the Di Francesco - Zuber diagrams [9] (one of the diagrams belonging to the original list was later removed by A. Ocneanu, very much like the tadpole graphs of $s l(2))$.

Several $\operatorname{sl}(3)$ quantum modules have self - fusion, namely: $\mathcal{A}_{k}$ itself, the $\mathcal{D}_{k}$ (when $k$ is divisible by 3 ), whose McKay diagrams are $\mathbb{Z}_{3}$ orbifolds of those of $\mathcal{A}_{k}$, and three exceptional cases called $\mathcal{E}_{5}, \mathcal{E}_{9}$ and $\mathcal{E}_{21}$, at levels 5, 9 and 21. The other modules (without self-fusion) are: the conjugated series $\mathcal{A}_{k}^{c}$, for which the number of simple objects is equal to the number of self-dual simple objects in $\mathcal{A}_{k}$, the $\mathcal{D}_{k}$ series, when $k=1$ or $2 \bmod 3$, the conjugated series $\mathcal{D}_{k}^{c}$, for all $k$, two modules of exceptionals called $\mathcal{E}_{5} / 3$, $\mathcal{E}_{9} / 3$, and finally the exceptional case $\mathcal{D}_{9}^{t}$ (a generalization of $E_{7}$ that can be obtained from $\mathcal{D}_{9}$ by using an exceptional twist), along with a "conjugated case" called $\mathcal{D}_{9}{ }^{t c}$. Some of the graphs of that system have double lines, like $\mathcal{E}_{9}$, so that it is not appropriate to say that they are "simply laced": better to consider them as "higher ADE". In all cases, with self-fusion or not, the rigidity property implied by $\mathcal{A}_{k}$ holds (the condition $\left(F_{\bar{n}}\right)_{a b}=\left(F_{n}\right)_{b a}$ does not forbid double lines). A new feature that appears in the $S U(3)$ situations (compared with $S U(2)$ ) is that one can sometimes find several graphs, and therefore several module-categories, with the same partition function. The quantum modules of $S U(3)$, arising when $q^{3+k}=-1$, are related as follows with conformal embeddings (we use a notation where the level $k$ appears as a subscript, and a quantum module at level $k$ is always a module over $\mathcal{A}_{k}$ ): The $\mathcal{A}_{k}(S U(3))$ are obtained by truncation of the Weyl chamber of $S U(3)$ at level $k$. $\mathcal{E}_{5}(S U(3)), \mathcal{E}_{9}(S U(3))$ and $\mathcal{E}_{21}(S U(3))$ are exceptional quantum subgroups obtained from the conformal embedding of $S U(3)_{5}$ in $S U(6)$, of $S U(3)_{9}$ in $E_{6}$ and of $S U(3)_{21}$ in $E_{7}$. Quantum modules of type $\mathcal{D}_{k}(S U(3))$ are obtained from non-simple conformal embeddings of $S U(3)_{k} \times S U(k)_{3}$ in $S U(3 k)$ (giving rise to quantum subgroups of $S U(3) \times S U(k)$ ) followed 
by contraction with respect to $S U(k)$. As recalled previously, we have also a twisted exceptional case $\mathcal{D}_{9}{ }^{t c}$. The smallest member $\mathcal{D}_{3}(S U(3))$ of the series with self-fusion $\mathcal{D}_{3 s}(S U(3))$ can be obtained directly from a conformal embedding of $S U(3)_{3}$ in $\operatorname{Spin}(8)$. If $\mathcal{M}$ denotes some modular invariant of $S U(3)$ type, and if $\mathfrak{C}$ denotes the permutation matrix describing complex conjugation on the set of irreducible representations of $\mathcal{A}_{k}(S U(3))$, one obtains another modular invariant (sometimes the same) by taking $\mathcal{M}^{\mathfrak{c}}=\mathcal{M} \mathfrak{C}$. Starting from $\mathcal{A}_{k}, \mathcal{D}_{k}, \mathcal{E}_{5}$ or $\mathcal{E}_{9}$, one builds in this way the conjugated series and graphs $\mathcal{A}_{k}{ }^{\mathfrak{c}}, \mathcal{D}_{k}{ }^{\mathfrak{c}}, \mathcal{E}_{5}{ }^{c}$ and $\mathcal{E}_{9}{ }^{c}$.

$S U(4)$. In this case, the classification of modular invariant partition functions was not a priori known, but people knew a list of examples. From this list and using modular splitting techniques - see a later section - the classification of $S U(4)$ Cayley graphs associated with WZW models, i.e., the classification of quantum modules of type $S U(4)$, was completed by A. Ocneanu and presented in [24]. The $S U(4)$ family includes the $\mathcal{A}_{k}(S U(4))$ series and its conjugate for all $k$, two kinds of orbifolds, the $\mathcal{D}_{k}^{(2)}=\mathcal{A}_{k} / 2$ series for all $k$ (with self-fusion when $k$ is even) and the $\mathcal{D}_{k}^{(4)}=\mathcal{A}_{k} / 4$ series for $k=0,2,6 \bmod 8$ (with self-fusion when $k$ is divisible by 8 ), their corresponding conjugated series, one exceptional case obtained by twisting, $\mathcal{D}_{8}^{(4) t}$, without self-fusion (a generalization of $E_{7}$ ) and finally three exceptional quantum graphs with self-fusion, at levels 4, 6 and 8, denoted $\mathcal{E}_{4}, \mathcal{E}_{6}$ and $\mathcal{E}_{8}$, together with one exceptional module for each of the last two. These exceptional quantum subgroups at levels $4,6,8$ can be respectively obtained from the study of the conformal embeddings of $S U(4)$ into $\operatorname{Spin}(15), S U(10)$ and $\operatorname{Spin}(20)$, see [7]; their modules (for the last two, since $\mathcal{E}_{4}$ doesn't have any) are obtained as a by-product of the determination of their algebras of quantum symmetries. We should also mention the existence of a conformal embedding of $S U(4)$, at level 2, in $S U(6)$, but it gives rise to $\mathcal{D}_{2}^{(2)}=\mathcal{A}_{2} / 2$, the first member of the $\mathcal{D}_{k}^{(2)}$ series, which can itself be obtained by conformal embedding of the non simple $S U(4) \times S U(k)$ in $S U(4 k)$, followed by reduction.

2.2. Quantum modules for general Lie groups $K$. To every quantum module (or quantum subgroup) one can associate a modular invariant partition function, but on general grounds, one would not hope that this information alone could be sufficient to reverse the machine. In any case, one could immediately object that the list of all modular invariant partition functions of type $K$ (an arbitrary Lie group, that we may assume to be simple or semi-simple) is not known. Such an argument seems to indicate that a general classification is out of reach. One could nevertheless point out the fact that classifying modular invariant partition functions does not 
coincide exactly with the problem of classifying quantum modules of a given Lie group $K$. For this later problem, it appears that the situation is not as bad as it may seem, because of the important role played by those quantum modules that are related to conformal embeddings (more about it later).

The fact that a given quantum module of type $K$ is also a quantum subgroup (existence of self-fusion) was often considered, in the past, as a curiosity. However, a detailed analysis of all known quantum modules indicates that quantum subgroups play a prominent role in the sense that all known cases, even if they do not possess self-fusion, can be somehow deduced from the quantum subgroups themselves. One possibility is to start from a quantum subgroup of a non simple Lie group $K$, and then perform reduction possibly followed by charge conjugation or twisting. Another possibility arises as follows: one starts from a conformal embedding, and obtains the associated modular invariant partition function. Then, solving modular splitting equations leads to an Ocneanu graph, and by construction the span of left (or right) generators build a graph with self-fusion that gives rise to a quantum subgroup, but in some cases a quantum module appears in the Ocneanu graph (it can be associated with the same partition function as the quantum subgroup we started with, or not). Warning: in the cases obtained by (direct) conformal embedding, the Ocneanu graph contains the McKay graph, but this property does not always hold for more general cases, for instance the $D_{\text {odd }}$ cases of $S U(2)$ have graphs of quantum symmetries that can be identified with Dynkin diagrams of type $A$ and therefore do not contain components of type $D$.

Although we have no proof that any quantum subgroup or quantum module of a given type (say $K$ ) can be obtained in one of these ways (direct or "indirect" conformal embedding) we are not aware of a single exception and it is tempting to conjecture that this is always so. Then, classifying quantum subgroups of type $K$ (and therefore classifying their associated modular invariants) would be achieved first by analyzing all possible conformal embeddings of $\tilde{K}$ in some larger $G$, with $\tilde{K}$ a semi-simple (maybe reductive) Lie group containing $K$ as a simple factor, then studying quantum symmetries and performing contraction or using conjugation and twisting on the resulting component graphs.

\section{General methods}

The methods that we describe now, at least at the beginning of this section, can be used in full generality as soon as we have a modular invariant partition function to start with. However, some properties will only hold in those cases where we actually started from a conformal embedding. To simplify the exposition, and because it is anyway in the spirit of this article, this is what we suppose from now on.

São Paulo J.Math.Sci. 3, 2 (2009), 241-264 
Expressions for representatives of the generators $s$ and $t$ of the modular group, can be obtained from the Kac-Peterson formula [18] for any Lie group and for any level. Using then the Verlinde formula [29] (generalizing the $S U(2)$ Hurwitz formula [17]) one can obtain matrix representatives for all possible fusion matrices. Practically, the most efficient method is to determine first the fusion matrices $N_{f}$ associated with fundamental representations of $K$ and then to use known recursion formulae (representation theory of $K$ ) to determine the others. The ring $\mathcal{A}_{k}(K)$ is therefore totally determined at this step.

We assume that we are given some chosen conformal embedding of $K$ at level $k$ (i.e., for a root of unity $q$ with $q^{g_{K}+k}=-1$ ), where $g_{K}$ is the dual Coxeter number, into a larger group $G$ at level 1 (i.e., for a root of unity $q$ with $q^{g_{G}+1}=-1$ ). From this conformal embedding, one determines the modular invariant $\mathcal{M}$, which is a matrix $r_{A} \times r_{A}$, where $r_{A}$ is the number of simple objects of $\mathcal{A}_{k}(K)$. One obtains immediately the dimensions $r_{E}=$ $\operatorname{Tr}(\mathcal{M})$ of $\mathcal{E}$ and $r_{O}=\operatorname{Tr}\left(\mathcal{M}^{\dagger} \mathcal{M}\right)$ of $\mathcal{O}$. It is also useful to determine the number $r_{W}$ of independent toric matrices, which is equal to the number of non-zero matrix elements of $\mathcal{M}$ (this number may be smaller than $r_{O}$ ).

In order to proceed, there are two possible roads. One is to determine first the algebra $\mathcal{O}$ of quantum symmetries and obtain the graph of $\mathcal{E}$ as a by-product (we are assuming self-fusion since we started from a conformal embedding). This is a two step process: one has first to solve an equation called "modular splitting equation" (see below) leading to a determination of all toric matrices of type $W_{x 0}$, of size $r_{A} \times r_{A}$, and then to determine the fundamental generators $O_{f}$ of $\mathcal{O}$ (of size $r_{O} \times r_{O}$ ) by solving a set of intertwining equations (see later), there are two such generators, called left and right, for each fundamental representation of $K$. The second possible road is to bypass the determination of $\mathcal{O}$ by solving the so-called "chiral modular splitting equation" (see below), this leads directly to the determination of $\mathcal{E}$, this is technically shorter that the first method, but it provides of course less information.

The modular splitting equation is a consequence of the module compatibility equation expressing $\mathcal{O}$ as an $\mathcal{A}_{k}$ bimodule :

$(m(n x p) q)=(m n) x(p q)$. It is obtained by writing this equation in terms of fusion matrices and toric matrices, and then choosing $x=0$. Explicitly, for each choice of the pair $(m, n)$ (i.e., $r_{A}^{2}$ possibilities), this equation reads: 


$$
N_{m} \mathcal{M} N_{n}^{t r}=\sum_{x=0}^{r_{O}-1}\left(W_{0 x}\right)_{m n} W_{x 0} .
$$

The left hand side involves only known fusion matrices and the modular invariant $\left(\mathcal{M}\right.$ is identified with $\left.W_{00}\right)$, whereas the right hand side is interpreted as a decomposition of the left hand side on a family (not always a base when $r_{W}<r_{O}$ ) of toric matrices $W_{x 0}$ to be determined. Remember that matrix elements of these toric matrices are non-negative integers. This equation is huge (the left hand side can be seen as an array with $r_{A}^{4}$ components) but it can be solved - i.e., one can find the $W_{x 0}$ thank's to a variety of techniques that will not be described here.

The quantum symmetry generators $O_{f}{ }^{L, R}$, where $f$ refers to the three fundamental representations of $K$, are then obtained by solving the intertwining equations:

$$
\begin{gathered}
N_{f} W_{x 0} N_{0}^{t r}=\sum_{y}\left(O_{f} L\right)_{x y} W_{y 0} \quad \text { and } \\
N_{0} W_{x 0} N_{f}^{t r}=\sum_{y}\left(O_{f R}\right)_{x y} W_{y 0}
\end{gathered}
$$

These are linear equations and the left hand side is known from the previous study, but one could a priori expect many solutions, however, taking into account the fact that matrix elements of $O_{f}$ are non-negative integers, and using several other properties (for instance the fact that these generators should obey polynomial equations expressing the vanishing of irreducible representations of level higher than $k$ ), one usually finds a unique solution, up to permutation matrices expressing the existence of possible isomorphisms for the Ocneanu graph $\mathcal{O}$, which is actually a collection of graphs (two for each fundamental generator of $K$ ) that can, themselves, be nonconnected. Matrices $O_{f}{ }^{L, R}$, obtained at the end of the last step, are the adjacency matrices of $\mathcal{O}$. These generators are "fundamental" in the sense that all other linear generators of $\mathcal{O}$ appear when we decompose products of these elements. The graph $\mathcal{O}$, which is the Cayley graph of multiplication by these fundamental generators, has a left and a right part (they are isomorphic), respectively associated with $O_{f}{ }^{L}$ and $O_{f}{ }^{R}$, but each part usually decomposes as a union of disconnected components, because of the existence of modules. Left and right chiral subalgebras are defined as the particular modules containing the identity element of $\mathcal{O}$. The graph $\mathcal{E}$ itself is also a union of graphs, one for each $f$, and it describes multiplication 
within these (isomorphic) chiral subalgebras; existence of self-fusion is, in our case ${ }^{3}$, automatic since $\mathcal{E}$ is recovered as chiral subalgebra of $\mathcal{O}$.

The "chiral method" that we summarize now is technically shorter that the first (the general one) since it uses only the first line of the modular invariant matrix and involves only $r_{A}^{2}$ equations rather than $r_{A}^{4}$, but it also gives less information. For instance one cannot discover the possible existence of quantum modules from the structure of the algebra of quantum symmetries, since the later is not obtained from this simplified method. The chiral modular splitting equation is a consequence of the module compatibility equation expressing $\mathcal{E}$ as an $\mathcal{A}_{k}$ module: $((m n) a)=(m(n(a)))$, where $m$ and $n$ are vertices of $\mathcal{A}_{k}$ and $a$ is a vertex of $\mathcal{E}$. This is immediately translated in terms of a relation between annular and fusion matrices. A particular case reads $\sum_{p} N_{m n p} F_{p_{00}}=\sum_{b} F_{n 0 b} F_{m b 0}$. The final ingredient is that, for a quantum subgroup $\mathcal{E}$ that can be identified with a chiral subalgebra of $\mathcal{O}$, property that holds in the present case, $\left(F_{p}\right)_{00}$ coincides $^{4}$ with the first line $\left(W_{00}\right)_{(p 0)}=\mathcal{M}_{p 0}$ of the modular matrix. The above equation, whose left hand side, that we call $\mathcal{K}_{m n}=\sum_{p} N_{m n p} \mathcal{M}_{p 0}$, is known, should then be solved over non-negative integers, for all $m, n$, leading to a determination of the rectangular matrix $E_{0}=\left(E_{0}\right)_{n b}=\left(F_{n}\right)_{0 b}$ sometimes called essential intertwiner [4]. The main fact that allows one to solve this set of equations is the following observation: choose a scalar product in the vector space $\mathcal{E}$ for which the simple generators $a$ are orthonormal and consider the vector $\mathcal{K}_{n}=\sum_{b}\left(F_{n}\right)_{0 b} b \in \mathcal{E}$. Because of the fact that the chiral modular splitting equation should be obeyed, the norm of this vector, equal to $\sum_{b}\left|\left(F_{n}\right)_{0 b}\right|^{2}=\sum_{b}\left(F_{n}\right)_{0 b}\left(F_{\bar{n}}\right)_{b 0}$ can be read from the expression of the known matrix $\mathcal{K}:\left\|\mathcal{K}_{n}\right\|=\mathcal{K}_{\bar{n} n}$. Because coefficients are non negative integers this information is usually sufficient to determine the different columns ("essential vectors") of the rectangular matrix $E_{0}$. Practically, for each choice of $m$ we calculate the vector $\mathcal{K}_{m}$ and determine its norm as indicated. Those of norm 1 already define columns of $E_{0}$; in particular one recovers $\left(E_{0}\right)_{p 0}=(\mathcal{M})_{p 0}$ as the first column. Then we consider vectors $\mathcal{K}_{m}$ of increasing norms $1,2,3 \ldots$ The process ultimately stops since the rank is finite. A complication, leading to ambiguities in the decomposition of $\mathcal{K}_{m n}$, stems from the fact that the family made of the different columns of $E_{0}$ is usually not free (the rank of the matrix $\mathcal{K}$, with matrix elements $\mathcal{K}_{m n}$ may be smaller than $r_{E}$ ).

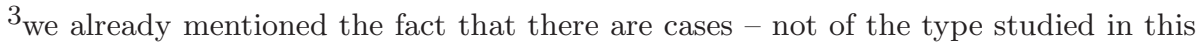
section - where $\mathcal{E}$ cannot be identified with a component of $\mathcal{O}$.

${ }^{4}$ For modular invariants $\mathcal{M}$ obtained by conformal embedding one can identify the first column of $E_{0}$ with the first column of $\mathcal{M}$ but it is not so in other cases, although one can always recover the full invariant $\mathcal{M}$ as the first toric matrix $W_{00}$.
} 
Once $E_{0}$ is known, the next and final step is to solve a simplified version of the intertwining equations, namely to find $G_{f}$, the adjacency matrix $G_{f}$ of the quantum graph $\mathcal{E}$ for each fundamental generator $f$ of $K$ (so, there are as many adjacency matrices $G_{f}$ as the rank of $K$ ): it should be such that $N_{f} E_{0}=E_{0} G_{f}$, where $N_{f}$ is the fusion matrix of the fundamental representation $f$. Of course, in order to solve these equations, one has to use the fact that all matrix elements of the $G_{f}$ 's are non-negative integers. In the last section, we shall illustrate these techniques (the chiral method) on a particular example. The reader interested in seeing how the general machinery works (the general method using the full collection of modular splitting equations and the determination of the graph $\mathcal{O}$ ) is referred for instance to the non trivial examples studied in [7].

If the group $K$ is semi-simple rather than simple, there is standard method, called contraction, described for instance in [8], that allows one to obtain a modular invariant for a simple component of $K$, starting from an invariant of $K$. This is generally a two-step process: One starts from a non-simple conformal embedding $K=K_{1} \times K_{2} \subset G$ and a modular invariant of $G$ at level 1 (which is not necessarily diagonal), then uses the corresponding branching rules to obtain a modular invariant for $K$; finally one performs the contraction with respect to a modular invariant associated with a chosen component, say $K_{1}$, of $K$ (this invariant is not required to be diagonal either) and one obtains an invariant for $K_{2}$. This method, which leads to a quantum module of $K_{2}$, does not require the determination of the graph of the quantum subgroup of $K$ since it is enough to perform the contraction at the level of the invariant. Once we have the invariant for $K_{2}$, we may apply the above general techniques, but one should be warned that, in such a case, some properties may be lost, for instance self-fusion (the obtained quantum module may not be a quantum subgroup) and there is no guarantee, a priori, that the chiral modular splitting method can be applied, since one does not a priori know if the equality between $\mathcal{M}_{p 0}$ and $\left(E_{0}\right)_{p 0}$ holds.

\section{Quantum subgroups of Lie groups from conformal embed- dings}

4.1. General. All the pairs $(K \subset G)$ listed below, known as conformal pairs, were known more than twenty years ago (see [1], [27], [19]), the novelty in our presentation is only a change of perspective since, rather than listing conformal subalgebras of $G$, we are interested in quantum subgroups of $K$, generically denoted $\mathcal{E}_{k}(K), k$ being the level. Therefore we fix $K$ and look for "overgroups" $G$ such that the pair $(K, G)$ is conformal. The fact that each such pair gives rise to a quantum subgroup of $K$ results from investigations carried out more recently (in the last ten years) but 
we should stress than few of them have been worked out explicitly: only the $S U(N)$ cases with $N=2,3,4$ are described (their associated graphs and algebras of quantum symmetries are known) in the available literature $[23,4,26,24,5,14,15,6,7]$. We always assume that $G$ is simple. In the first part, we take $K$ is simple, and in the next, $K$ semi-simple, but non simple. Warning: because they require a special treatment, we do not mention the possible quantum subgroups arising from those conformal embeddings for which $K$ would possess a $U(1)$ factor. Finally, we also assume that those conformal embeddings are maximal since non maximal ones can be constructed by considering chains of inclusions of maximal embeddings, with the constraint that only the last one may have a Dynkin index not equal to one. Not all conformal embeddings $K \subset G$ correspond to isotropy-irreducible pairs (see [30] for the list of such pairs) and not all isotropy-irreducible homogeneous spaces (whether they are symmetric or not) define conformal embeddings, however the two lists almost coincide, this is why we mention below this property. Notice that an embedding ${ }^{5}$ $K \subset \operatorname{Spin}(\operatorname{dim}(K))$, at a level equal to the dual Coxeter number of $K$, is always conformal (it is called "adjoint", for obvious reasons).

For unitary cases, the so called rank-duality property states that whenever there exists a quantum subgroup of $S U(N)$ at level $k$, there exists also a quantum subgroup of $S U(k)$ at level $N$. The quantum subgroups that we describe in the next subsection are obtained by "direct" conformal embeddings (ie not followed by contraction). On general grounds, for every such example, one can also consider its rank-level dual. It may be that such a dual can itself be constructed from a direct conformal embedding (so it would appear in the list given next). When it is not so, the dual will result from from some non-simple conformal embedding (ie with a non simple $\mathrm{K}$ included into some G) followed by a contraction. Let us illustrate this with a simple example: at level 10 we have an exceptional $E_{6}$ invariant of $S U(2)$ from the inclusion of $S U(2)$ into $\operatorname{Spin}(5)$. Therefore we should have also an exceptional invariant of $S U(10)$ at level 2; the corresponding quantum subgroup $\mathcal{E}_{2}(S U(10))$ does not appear in the lists given in the next section, but it does exist, and its modular invariant can be obtained from the nonsimple conformal embedding $S U(2) \times S U(10) \subset S U(20)$, starting from the diagonal invariant of the later at level 1 followed by a contraction on the $E_{6}$ invariant of $S U(2)$.

\subsection{Quantum subgroups of simple Lie groups $K$.}

$S U(N)$. When $N \neq 2,3,4,6,8,9$ there are only three conformal embeddings, into $S U(N(N-1) / 2), \operatorname{Spin}\left(N^{2}-1\right)$ and $S U(N(N+1) / 2)$; they are

\footnotetext{
${ }^{5}$ In what follows, we shall usually write $S O(N)$ for the orthogonal group, with the understanding that, in most cases, one should instead consider its universal cover $\operatorname{Spin}(N)$.
} 
called antisymmetric, adjoint, and symmetric, and they occur at respective levels $k$ equal to $N-2, N$ and $N+2$. The corresponding exceptional quantum subgroups are therefore denoted $\mathcal{E}_{N-2}(S U(N)), \mathcal{E}_{N}(S U(N))$ and $\mathcal{E}_{N+2}(S U(N))$. For small $N$, one of them may coincide with the smallest member of some $\mathcal{D}$ series.

When $N=2$, only the standard case $k=N+2$ is non trivial (giving rise to $\left.D_{4}=\mathcal{D}_{4}(S U(2))\right)$, but we have also very exceptional embeddings into $\operatorname{Spin}(5)$ (giving rise to $\left.E_{6}=\mathcal{E}_{10}(S U(2))\right)$ and into $G_{2}$ giving rise to $\left.E_{8}=\mathcal{E}_{28}(S U(2))\right)$.

When $N=3$, only the standard cases $k=N$ (giving rise to $\mathcal{D}_{3}(S U(3))$ ) and $k=N+2$ (giving rise to $\mathcal{E}_{5}(S U(3))$ ) are non trivial, but we have also two very exceptional embeddings into $E_{6}$ (giving rise to $\mathcal{E}_{9}(S U(3))$ ) and into $E_{7}$ (giving rise to $\mathcal{E}_{21}(S U(3))$ ).

When $N=4$, the three standard cases do exist and give rise respectively to $\mathcal{D}_{2}(S U(4)), \mathcal{E}_{4}(S U(4))$ and $\mathcal{E}_{6}(S U(4))$, but we have a very exceptional embedding into $\operatorname{Spin}(20)$ giving rise to $\mathcal{E}_{8}(S U(4))$. The later is actually the smallest member of a series existing only for $S O(2 N)$ groups (but we know that $S U(4) \simeq S O(6))$.

When $N=6$, the three standard cases exist and give rise to $\mathcal{E}_{4}(S U(6))$, $\mathcal{E}_{6}(S U(6))$ and $\mathcal{E}_{8}(S U(6))$, but there is a very exceptional embedding into $S p(10)$ giving rise to another exceptional quantum subgroup at level 6: $\mathcal{E}^{\prime}{ }_{6}(S U(6))$.

When $N=8$, the three standard cases exist and give rise to $\mathcal{E}_{6}(S U(8))$, $\mathcal{E}_{8}(S U(8))$ and $\mathcal{E}_{10}(S U(8))$, but there are two very exceptional embedding: one into $E_{7}$ giving rise to $\mathcal{E}_{1}(S U(8))$, and one into $S O(70)$ giving rise to another $\mathcal{E}^{\prime}{ }_{10}(S U(8))$ at level 10.

When $N=9$, on top of the three standard cases $\mathcal{E}_{7}(S U(9)), \mathcal{E}_{9}(S U(9))$ and $\mathcal{E}_{11}(S U(9))$, we have a very exceptional quantum subgroup $\mathcal{E}_{1}(S U(9))$ coming from an embedding into $E_{8}$.

In all cases, one can construct the associated rank-level dual quantum subgroups (see our comment in the last section).

$S O(2 N)$. When $2 N \neq 6,10,12,16$ there are only three conformal embeddings: into $S U(2 N)$, for $k=2$, into $S O\left(2 N^{2}-N\right)$, for $k=2 N-2$ and into $S O\left(2 N^{2}+N-1\right)$, for $k=2 N+2$. The case $N=6$ coincides with the case $S U(4)$. For $N=10,12,16$, on top of these standard exceptional cases, we have non standard quantum subgroups $\mathcal{E}_{4}(S O(10))$ coming from an embedding into $S U(16), \mathcal{E}_{8}(S O(12))$ coming from an embedding into $S p(16)$, and two other exceptional cases for $S O(16)$ : $\mathcal{E}_{1}(S O(16))$ coming from an embedding into $E_{8}$ and $\mathcal{E}_{16}(S O(16))$ coming from an embedding into $S O(128)$. 
$S O(2 N+1)$. When $2 N+1 \neq 3,5,9$ there are only three conformal embeddings: into $S U(2 N+1)$ for $k=2$, the adjoint embedding into $S O(N(2 N+$ 1)) at level $k=2 N-1$, and a last one into $S O(N(2 N+3))$ for $k=2 N+3$. The particular case $S O(3)$ coincides with $S U(2)$. The particular case $S O(5)$ coincides with $S p(2)$ (see next entry), so here we have the three quantum subgroups arising from conformal embeddings into $S U(5), S O(10)$ and $S O(14)$, but there is also an embedding of $S O(5) \subset E_{8}$ giving rise to an exceptional $\mathcal{E}_{12}(S O(5))$ which is the only one in the whole list (with $K$ simple) for which the space $G / K$ is not isotropy irreducible. Finally $S O(9)$, on top of its three standard exceptional quantum subgroups, has a non standard $\mathcal{E}_{2}(S O(9))$ coming from its embedding into $S O(16)$.

$S p(N)$. When ${ }^{6} N \neq 2,3,4$ we have only the adjoint embedding into $S O(N(2 N+1))$, at level $N+1$, and also an embedding into $S O(N(2 N+1))$ at level $N-1$. The case $N=2$ coincides with $S O(5)$ so that we have conformal embeddings into $S U(5), S O(10), S O(14)$ and $E_{8}$ at respective levels $2,3,7$ and 12 . When $N=3$, we have also a non standard exceptional $\mathcal{E}_{5}(S p(3))$ coming from an embedding into $S p(7)$, and when $N=4$ one obtains two other quantum subgroups : $\mathcal{E}_{1}(S p(4))$ from an embedding into $E_{6}$, and $\mathcal{E}_{7}(S p(4))$ from an embedding into $S O(42)$.

$E_{6}$. One finds $\mathcal{E}_{6}\left(E_{6}\right)$ coming from an embedding into $S U(27)$, and $\mathcal{E}_{12}\left(E_{6}\right)$ coming from the adjoint embedding into $\operatorname{Spin}(78)$.

$E_{7}$. One finds $\mathcal{E}_{12}\left(E_{7}\right)$ coming from an embedding into $S p(28)$, and $\mathcal{E}_{18}\left(E_{7}\right)$ coming from the adjoint embedding into $\operatorname{Spin}(133)$.

$E_{8}$. We have only $\mathcal{E}_{30}\left(E_{8}\right)$ coming from the adjoint embedding into $\operatorname{Spin}(248)$.

$F_{4}$. One finds $\mathcal{E}_{3}\left(F_{4}\right)$ coming from an embedding into $\operatorname{Spin}(26)$, and $\mathcal{E}_{9}\left(F_{4}\right)$ coming from the adjoint embedding into $\operatorname{Spin}(52)$.

$G_{2}$. One finds $\mathcal{E}_{3}\left(G_{2}\right)$ coming from an embedding into $E_{6}$, and $\mathcal{E}_{4}\left(G_{2}\right)$ coming from the adjoint embedding into $\operatorname{Spin}(14)$.

4.3. Quantum subgroups of semi-simple but non-simple Lie groups $K$. Each of the following conformal embeddings, of the type $K \subset G$, gives rise to a quantum subgroup of the semi-simple (but not simple) group $K$. After reduction (diagonal or not) with respect to some factor, one obtains a quantum module (not always a quantum subgroup) of the remaining factor. Many of them can be obtained in more than one way. Like in [1] we list separately those cases corresponding to isotropy irreducible pairs $(K, G)$ but the general analysis is the same.

\footnotetext{
${ }^{6}$ Warning : $S p(N)$ is sometimes denoted $S p(2 N)$ in the literature.
} 
Cases arising from isotropy irreducible conformal embeddings. $S U(2) \times$ $E_{7} \subset E_{8}(k=(1,1)), S U(2) \times F_{4} \subset E_{7}(k=(3,1)), S U(2) \times S U(6) \subset$ $E_{6}(k=(1,1)), S U(2) \times S O(12) \subset E_{7},(k=(1,1)), S U(2) \times S p(3) \subset$ $F_{4}(k=(1,1)), S U(2) \times S U(2) \subset G_{2}(k=(1,3)), S U(2) \times G_{2} \subset F_{4}(k=$ $(8,1)), S U(3) \times G_{2} \subset E_{6}(k=(2,1)), S U(3) \times S U(6) \subset E_{7}(k=(1,1))$, $S U(3) \times S U(3) \subset F_{4}(k=(1,2)), S U(3) \times S U(3) \times S U(3) \subset E_{6}(k=$ $(1,1,1)), S U(3) \times E_{6} \subset E_{8}(k=(1,1)), G_{2} \times S p(3) \subset E_{7}(k=(1,1))$, $F_{4} \times G_{2} \subset E_{8}(k=(1,1))$. We have also the series $S U(2) \times S O(q) \subset$ $S p(q)(k=(q, 4))$ and $S U(p) \times S U(q) \subset S U(p q)(k=(q, p)$, and $S O(p) \times$ $S O(q) \subset S O(p+q)$, with $k=(1,1)$, that also exists for $p=4$ with $S O(4) \simeq S U(2) \times S U(2))$.

Non isotropy irreducible conformal embeddings. $S O(p) \times S O(q) \subset S O(p q)$, $k=(q, p)$ (which also exists when $p=4$, with $S O(4) \simeq S U(2) \times S U(2)$ ), $S p(p) \times S p(q) \subset S O(4 p q), k=(q, p), S U(5) \times S U(5) \subset E_{8}, k=(1,1)$, $S U(2) \times G_{2} \subset E_{7}, k=(7,2)$, and finally $S U(2) \times S U(3) \subset E_{8}, k=(16,6)$ that we choose below as an example.

\section{Example of a quantum subgroup of $S U(2) \times S U(3)$}

The homogenous space $G / K$. Take $K=S U(2) \times S U(3) \subset G=E_{8}$. This embedding is maximal, but the space $G / K$ is not symmetric and is not isotropy-irreducible either. Indeed, reduction of the adjoint representation of $G$ reads $^{7}$ (the second bracket gives the decomposition of the isotropy representation) : $248 \mapsto[(3 \times 1)+(1 \times 8)]+[7 \times 8+5 \times 10+5 \times \overline{10}+3 \times 27]$.

The Dynkin index of the representations. The index of the adjoint representation of a Lie group coincides with its dual Coxeter number, so we obtain immediately $I_{E_{8}}=30, I_{S U(2)}=2, I_{S U(3)}=3$. For the other representations, one has to use the following: The Dynkin index $I_{\lambda}$ of a representation $\lambda$ of a Lie group $K$ is obtained by the standard formula $I_{\lambda}=\frac{\operatorname{dim}(\lambda)}{2 \operatorname{dim}(K)}\langle\lambda, \lambda+2 \rho\rangle$ where $\rho$ is the Weyl vector and where $\langle$,$\rangle is the$ fundamental quadratic form (the inverse of the Cartan matrix). In the case of $S U(2), \rho=\{1\}$ and the quadratic form is $(1 / 2)$. In the case of $S U(3)$, $\rho=\{1,1\}$ and the quadratic form is $\frac{1}{3}\left(\begin{array}{ll}2 & 1 \\ 1 & 2\end{array}\right)$. One obtains in this way the following indices for the representations appearing in the previous branching rule:

$$
30 \mapsto[(2,0) \quad(0,3)],[(28,3) \quad(10,15 / 2) \quad(10,15 / 2) \quad(2,27)]
$$

\footnotetext{
7 irreducible representations are labeled by their dimensions since there is no ambiguity here.
} 
The Dynkin index of this embedding. The Dynkin index $k$ of an embedding $K \subset G$ defined by a branching rule $\mu \mapsto \sum_{j} \alpha_{j} \nu_{j}, \alpha_{j}$ being multiplicities, is given by

$$
k=\sum_{j} \alpha_{j} I_{\nu_{j}} / I_{\mu}
$$

where $I_{\mu}, I_{\nu} \ldots$ are quadratic Dynkin indices for the corresponding representations.

$$
\begin{aligned}
S U(2) & : \\
k_{1} & =\frac{1}{30}[1 \times 2+8 \times 0+8 \times 28+10 \times 10+10 \times 10+27 \times 2]=16 \\
S U(3) & : \\
k_{2} & =\frac{1}{30}[3 \times 0+1 \times 3+7 \times 3+5 \times 15 / 2+5 \times 15 / 2+3 \times 27]=6
\end{aligned}
$$

The Dynkin index of this embedding of $S U(2) \times S U(3)$ in $E_{8}$ is therefore $\left(k_{1}, k_{2}\right)=(16,6)$.

This embedding is conformal. Use $K_{1}=S U(2), K_{2}=S U(3), K=K_{1} \times$ $K_{2}, G=E_{8}$ to calculate the following quantities (central charges): $c_{1}=$ $\frac{\operatorname{dim}\left(K_{1}\right) \times k_{1}}{k_{1}+g_{K_{1}}}=(3 \times 16) /(16+2)=8 / 3, c_{2}=\frac{\operatorname{dim}\left(K_{2}\right) \times k_{2}}{k_{2}+g_{K_{2}}}=(8 \times 6) /(6+3)=$ $16 / 3, c_{K}=c_{1}+c_{2}=8$. This embedding of $S U(2)$ (level 16) times $S U(3)$ (level 6) times into $E_{8}$ (level 1 ) is conformal since the following identity is satisfied: $c_{K}=c_{G}$, indeed $c_{G}=\frac{\operatorname{dim}(G) \times 1}{1+g_{G}}=(248 \times 1) /(1+30)=248 / 31=8$. Fusion matrices of $S U(2) \times S U(3)$. The fusion matrices $N_{p}(S U(2))$ at level 16 are of dimension $17 \times 17$, since $r_{A}=k_{1}+1$ for the ring $\mathcal{A}_{k_{1}}(S U(2))$; they are obtained from the recurrence relation for $S U(2)$ representations (Tchebychef polynomials), using $N_{0}=I d_{17}$ and $N_{1}$, the adjacency matrix for the graph $A_{17}=\mathcal{A}_{16}(S U(2))$ associated with the fundamental weight $\{1\}$.

The fusion matrices $N_{p}(S U(3))$ at level 6 are of dimension $(7 \times 8) / 2 \times$ $(7 \times 8) / 2=28 \times 28$, since $r_{A}=\left(k_{2}+1\right)\left(k_{2}+2\right) / 2$ ! for the ring $\mathcal{A}_{k_{2}}(S U(3))$; they are obtained from the recurrence relation for $S U(3)$ representations, using $N_{0}=I d_{28}$ and $N_{10}$, the adjacency matrix for the graph $\mathcal{A}_{6}(S U(3))$ associated with the fundamental weight $\{10\}$ and $N_{01}=N_{10}{ }^{t r}$ associated with the fundamental weight $\{01\}$.

The fusion matrices $N_{p}(S U(2) \times S U(3))$ at level $k$ are therefore of dimension $(17 \times 28) \times(17 \times 28)=476 \times 476$ and are obtained by taking tensor product of fusion matrices of $S U(2)$ and $S U(3)$.

The quantum (or affine) branching rules. The unique integrable representation, with highest weight $\lambda$, at level 1 of $E_{8}$ is the identity representation. 
This can be seen for example by using the integrability condition $1 \geq\langle\lambda, \theta\rangle$, where $\theta$ is the highest root of the chosen Lie algebra. Alternatively, one may calculate the q-dimensions of the fundamental representations of $E_{8}$ at a root of unity $q$ and keep only those that do not vanish at level 1, i.e., when $q$ is specialized to the value $q=\exp (i \pi / \kappa)$, with $\kappa=g_{G}+1=31$.

To any irreducible representation $\lambda$ (with non-zero $q$-trace) of $G$ or of $K$, one associates a conformal weight defined by $h_{\lambda}=\frac{\langle\lambda, \lambda+2 \rho\rangle}{2(k+g)}$ where $g$ is the dual Coxeter number of the chosen Lie algebra, $k$ is the level (for $G$, one chooses $k=1$ ), $\rho$ is the Weyl vector (of $G$, or of $K$ ). One builds the list of irreducible objects $\lambda$ of $G$ at level 1 (here we have only one) and calculate their conformal weights $h_{\lambda}$; then, one builds the list of irreducible objects $\mu$ of $K$ at level $k$ and calculate their conformal weights $h_{\mu}$. A necessary - but not sufficient - condition for an (affine or quantum) branching from $\lambda$ to $\mu$ is that $h_{\mu}=h_{\lambda}+m$ for some non-negative integer $m$. One can establish in this way a list of candidates for the branching rules $\lambda \hookrightarrow \sum_{n} c_{n} \mu_{n}$, where $c_{n}$ are positive integers to be determined; there exist several techniques (that we shall not describe here) to determine the coefficients $c_{n}$ (some of them can be 0 ). The final result giving the quantum branching rule for the reduction of the identity representation of $E_{8}$ (with $q^{31}=-1$ ) with respect to $S U(2) \times S U(3)$, respectively taken at roots of unity $q_{1}, q_{2}$ with $q_{1}{ }^{18}=-1, q_{2}{ }^{9}=-1$, is as follows (we label the representations by their highest weights $):(00000000) \rightarrow\left(s_{0}+s_{16}\right) \otimes\left(t_{00}+t_{60}+t_{06}\right)+\left(s_{4}+s_{12}\right) \otimes$ $\left(t_{30}+t_{33}+t_{03}\right)+\left(s_{2}+s_{14}+2 s_{8}\right) \otimes\left(t_{22}\right)+\left(s_{6}+s_{10}\right) \otimes\left(t_{11}+t_{41}+t_{14}\right)$.

The modular invariant. We write the diagonal invariant of type $G=E_{8}$ as a sum $\sum_{e} \lambda_{\bar{e}} \lambda_{e}$. Here it contains only one term: the square of $\lambda_{e}=$ $\{00000000\}$. Its associated quantum graph is denoted $\mathcal{J}=\mathcal{A}_{1}\left(E_{8}\right)$ has only one vertex. Using the previous branching rule, we replace, in this expression, each $\lambda_{e}$ (here, only one) by the corresponding sum of representations for $K$. The modular invariant $\mathcal{M}$ of type $K$ that we are looking for is parametrized by $\mathcal{Z}=\sum_{e \in \mathcal{J}}\left(\sum_{n} c_{n}(\bar{e}) \mu_{n}(\bar{e})\right)\left(\sum_{n} c_{n}(e) \mu_{n}(e)\right)$. Using an obvious ordering, associated with increasing levels on the set of representations, one obtains immediately the associated matrix $\mathcal{M}$ by writing $\mathcal{Z}=\sum_{m, n} \mu_{m} \mathcal{M}_{m n} \bar{\mu}_{n}$. Its dimension is $476 \times 476$ but it is very sparse.

Solving the chiral modular splitting equations. There are 22 non-zero entries on the first line of $\mathcal{M}$ at positions $\{1,1\},\{17,1\},\{13,10\},\{5,10\}$, $\{1,28\},\{17,28\},\{11,5\},\{7,5\},\{11,20\},\{7,20\},\{15,13\},\{3,13\},\{9,13\}$, $\{9,13\},\{13,7\},\{5,7\},\{13,25\},\{5,25\},\{11,17\},\{7,17\},\{1,22\}$ and $\{17,22\}$. We calculate the tensor $\mathcal{K}=\sum_{p} N_{p}(S U(2) \times S U(3)) \mathcal{M}_{p 0}$. It is a (sparse) array $(\mathcal{K})_{m n}$ of dimension $476 \times 476$ with 33598 non-zero entries. Its rank, and therefore also the rank of the family of essential vectors is 21. However, the number of vertices of the McKay graph of this quantum 
subgroup $\mathcal{E}$ of $S U(2) \times S U(3)$ is $r_{E}=\operatorname{Tr}(\mathcal{M})=24>21$, therefore we have to obtain 476 annular matrices $F_{p}$ of dimension $24 \times 24$. As we shall see later, the 24 vertices of the graph decomposes into three particular subsets $(24=10+7+7)$ with respect to $S U(2)$ and into two particular subsets $(24=12+12)$ with respect to $S U(3)$.

There are 120 vectors $\mathcal{K}_{n}$ of norm 1 but there are many repetitions in this family: only 16 of them are unequal (and independent), at positions $1,2,4,11,12,14,20,21,22,29,40,48,57,85,141,197$. These independent vectors already give us the first 16 columns of the essential matrix $E_{0}$.

The family of vectors $\mathcal{K}_{n}$ of norm 2 (those that should be written as sums of 2 column vectors of the essential matrix $E_{0}$ ) contains 63 entries, and among them, only 9 are distinct, however only those at positions 39,96, 49, 104 and 225 will concern us. Indeed, a detailed analysis of the chiral modular splitting equation for this family implies that we should add the following 8 vectors to complete our list : $\left(\mathcal{K}_{39}+\mathcal{K}_{96}\right) / 3,\left((2 / 3) \mathcal{K}_{39}-\right.$ $\left.(1 / 3) \mathcal{K}_{96}\right),\left(-(1 / 3) \mathcal{K}_{39}+(2 / 3) \mathcal{K}_{96}\right),\left(\mathcal{K}_{49}+\mathcal{K}_{104}\right) / 3,\left((2 / 3) \mathcal{K}_{49}-(1 / 3) \mathcal{K}_{104}\right)$, $\left(-(1 / 3) \mathcal{K}_{49}+(2 / 3) \mathcal{K}_{104}\right)$, and $\mathcal{K}_{225} / 2, \mathcal{K}_{225} / 2$, which indeed appears twice in the list (components of $\mathcal{K}_{225}$ are all even integers). We build in this way the rectangular matrix $E_{0}$ with 476 lines, 24 columns, the rank of this family of column vectors is 21 , as expected. Despite of the appearance of non integer (and non positive) coefficients in the above linear combinations, the matrix elements (columns of $E_{0}$ ) are indeed non negative integers. The last step is to check that all chiral modular splitting equations are satisfied. The obtained rectangular matrix $E_{0}$, sometimes called "the intertwiner", describes the induction-restriction functor between $S U(2) \times S U(3)$ at level $(16,6)$ and its quantum subgroup $\mathcal{E}$ (the non-zero entries in its first column, which is also the first column of $\mathcal{M}$, show the existence of non-commutative analogs for the Klein polynomials that appear in the theory of binary polynomial subgroups of $S U(2))$.

Solving the chiral intertwining equations. These equations, determining the adjacency matrices $G_{\{1\}}, G_{\{10\}}$ and $G_{\{01\}}=\left(G_{\{01\}}\right)^{t r}$, of dimension $24 \times 24$, of the graphs, are:

$$
\begin{aligned}
N_{\{1\},\{00\}} \cdot E_{0}= & E_{0} \cdot\left(G_{\{1\}} \otimes \mathbb{1}_{28}\right) \\
N_{\{0\},\{10\}} \cdot E_{0}= & E_{0} \cdot\left(\mathbb{1}_{17} \otimes G_{\{10\}}\right), \\
\text { with } N_{\{\lambda\},\left\{\lambda_{1} \lambda_{2}\right\}}= & N_{\{0\},\{01\}} \otimes E_{0}=E_{0} \cdot\left(\mathbb{1}_{17} \otimes G_{\{01\}}\right)
\end{aligned}
$$

The solution (one has to take into account the fact that coefficients are non-negative integers, of course) reads as follows: 


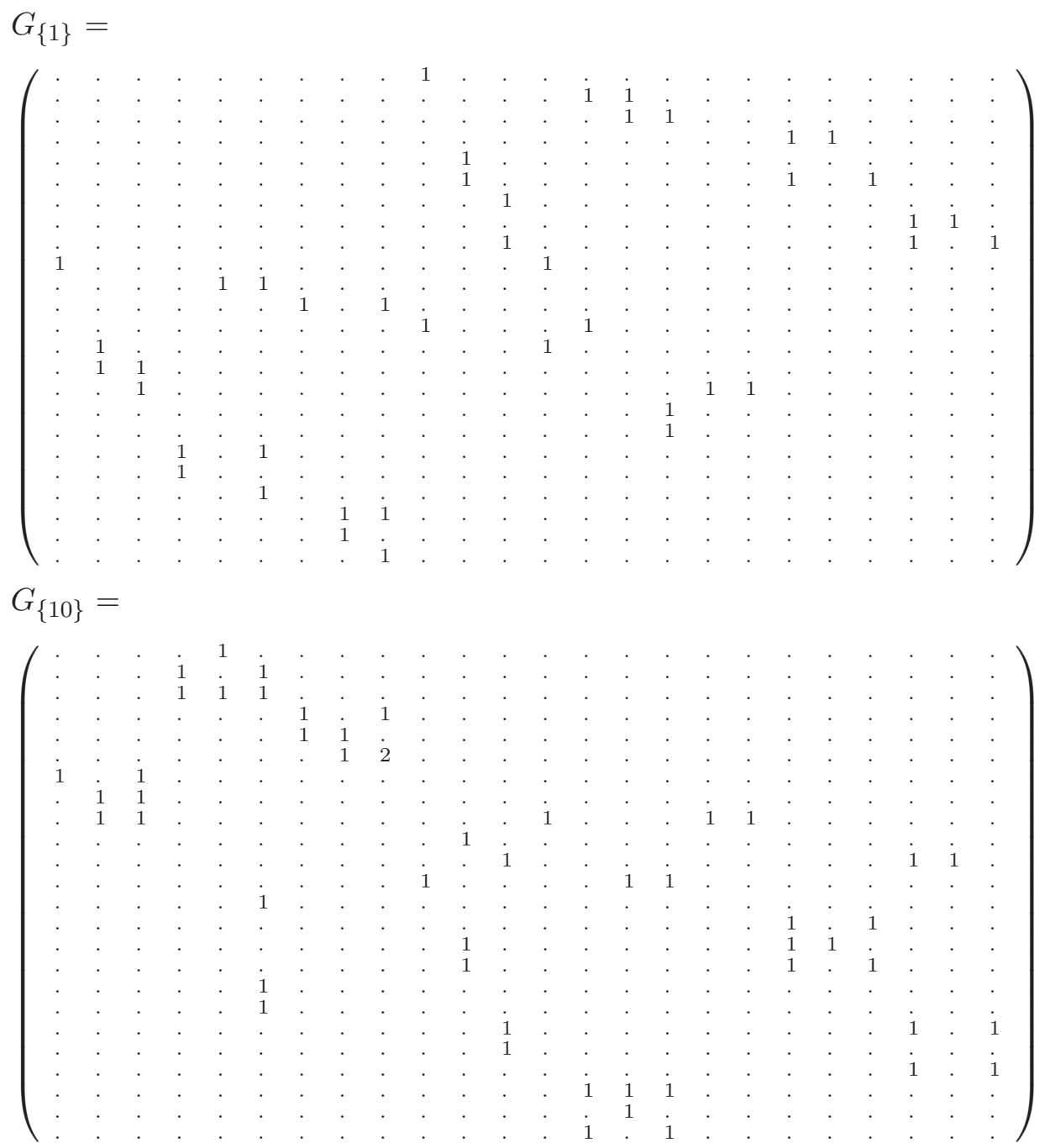

and $G_{01}=G_{10}{ }^{t r}$.

They are adjacency matrices for the following graphs : see figs 1 and 2 . The first is the union of three component graphs of $S U(2)$ type, namely the Dynkin diagrams $D_{10}$ and $E_{7}$ (twice), the other is the union of two components of $S U(3)$ type, namely $\mathcal{D}_{6}$ and its conjugated graph $\mathcal{D}_{6}(S U(3))^{\mathfrak{c}}$. The graph of $G_{01}$ is obtained from $G_{10}$ by reversing the arrows. Since the underlying sets of vertices are the same for $G_{1}$ and $G_{10}$ (they denote irreducible objects for this quantum subgroup of $S U(2) \times S U(3)$ ), it is better to draw them together, as on figure 3 . It is worth pointing out the 
fact that this later graph, with 24 vertices, has self-fusion (it represents a quantum subgroup of $S U(2) \times S U(3)$ !) whereas, it is not so for all its components, taken alone: $D_{10}=\mathcal{D}_{16}(S U(2))$ and $\mathcal{D}_{6}(S U(3))$ have self-fusion, but $E_{7}=\mathcal{E}_{16}(S U(2))$ and $\mathcal{D}_{6}(S U(3))^{\mathfrak{c}}$ don't.

Before ending this section, we should stress the fact that what we did here was to solve the chiral modular splitting equations (only) for this subgroups of $S U(2) \times S U(3)$. A full analysis of this example (that would determine toric matrices and the Ocneanu graph) would require to solve the full set of equations, not only the chiral part.

Using the conformal embedding of $S U(2) \times S U(3)$ in $E_{8}$, followed by contraction with respect to $S U(3)$, subtraction of the $D_{10}$ invariant of $S U(2)$ and division by 2 , is a standard way to obtain the $E_{7}$ invariant of $S U(2)$, see for instance [8]. This is therefore by no means a new result. The point that we want to make here is that the fact that $E_{7}$ and, for that matters, $\mathcal{D}_{6}(S U(3))^{\mathfrak{c}}$, do not have nice properties (no self-fusion, lack of "flatness" in subfactor terminology) is a result of the contraction process: if one does not perform contraction with respect to any of the two simple factors but just determine the McKay graph of the whole thing by using some of the general techniques sketched in previous pages, one obtains a "nice" quantum subgroup of $S U(2) \times S U(3)$. Instead of trying to classify and describe all quantum modules (and their modular invariant partition function) for a given simple Lie group $K$, it may be easier, and possibly more interesting, to consider only those that indeed define quantum subgroups (self-fusion), while at the same time relaxing the simplicity hypothesis in the choice of $K$. 

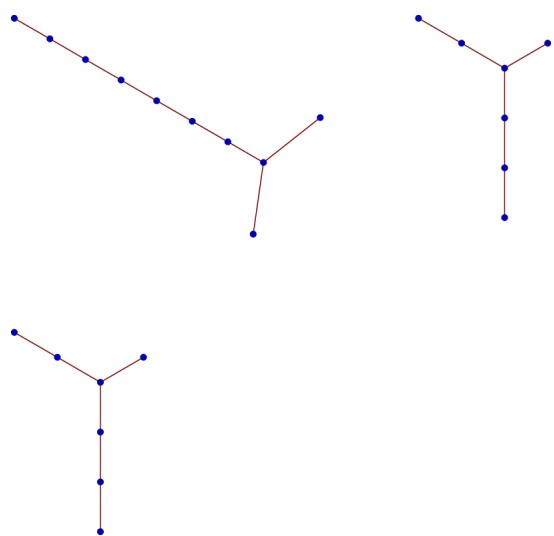

Figure 1. The graph of $G_{1}$. One recognizes the Dynkin diagrams $D_{10}$, and $E_{7}$, that appears twice. They describe the quantum subgroup $\mathcal{D}_{16}(S U(2))$ and two copies of the quantum module $\mathcal{E}_{16}(S U(2))$.
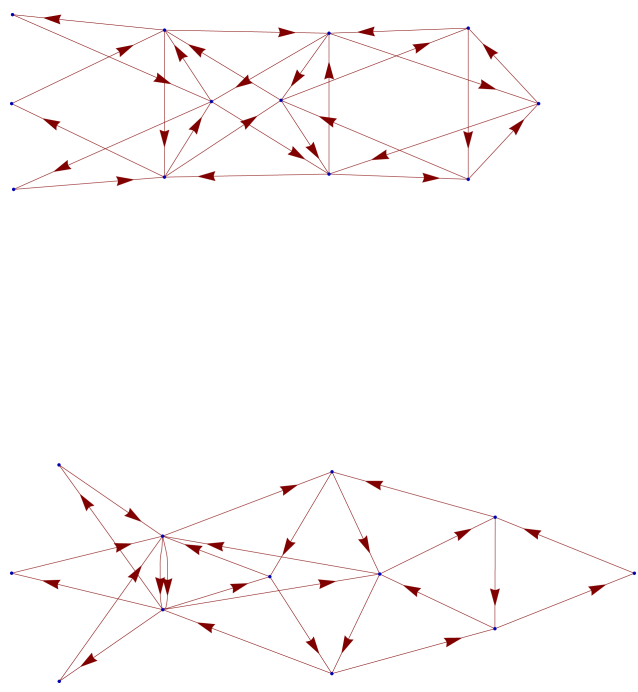

Figure 2. The graph of $G_{10}$. One recognizes the graphs of the quantum subgroup $\mathcal{D}_{6}(S U(3))$ (displayed on top, with the origin as its rightmost vertex), and its conjugate, the quantum module $\mathcal{D}_{6}(S U(3))^{\mathfrak{c}}$. 


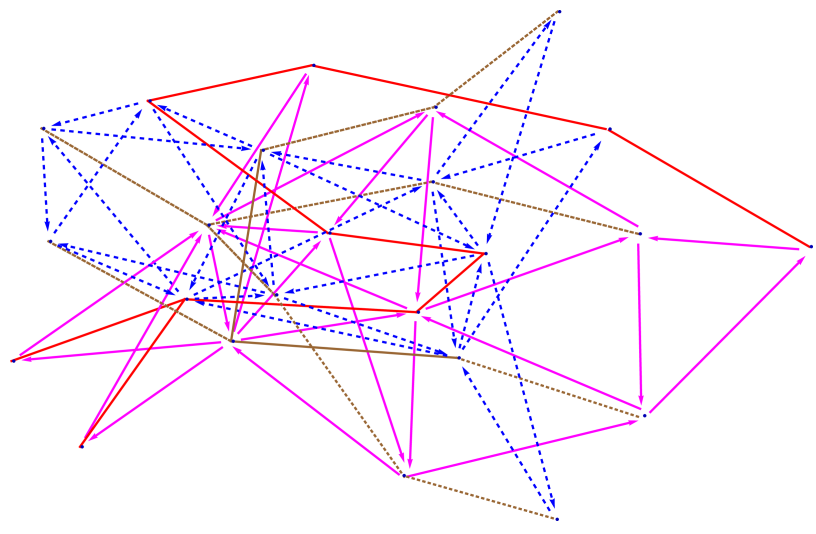

FiguRE 3. The graph of a quantum subgroup of $S U(2) \times$ $S U(3)$. The two subgraphs of type $E_{7}=\mathcal{E}_{16}(S U(2))$ appear with dotted lines (brown), the subgraph $D_{10}=\mathcal{D}_{16}(S U(2))$ with a plain line (red), the subgraph $\mathcal{D}_{6}(S U(3))$ is oriented and plain (magenta), the subgraph $\mathcal{D}_{6}{ }^{c}(S U(3))$ is oriented and dotted (blue). One of the edges of the later is double, see the second component of fig. 2. The origin is located on the rightmost vertex.

\section{References}

[1] Bais F and Bouwknegt P 1987 A classification of subgroup truncations of the bosonic string Nucl. Phys. B 279 pp 561-570.

[2] Böckenhauer J , Evans D and Kawahigashi Y 2000 Chiral structure of modular invariants for subfactors Commun. Math. Phys. 210 pp 733-784.

[3] Cappelli A, Itzykson C and Zuber J -B 1987 The ADE classification of minimal and $A_{1}^{(1)}$ conformal invariant theories Commun. Math. Phys. 13 p 1.

[4] Coquereaux R 2002 Notes on the quantum tetrahedron Moscow Math. J. 2 no 1 pp 1-40 (preprint hep-th/0011006).

[5] Coquereaux R and Schieber G 2002 Twisted partition functions for $A D E$ boundary conformal field theories and Ocneanu algebras of quantum symmetries $J$. of Geom. and Phys. 781 pp 1-43 (preprint hep-th/0107001).

[6] Coquereaux R and Schieber G 2007 Orders and dimensions for $\mathrm{sl}(2)$ or $\mathrm{sl}(3)$ module categories and Boundary Conformal Field Theories on a torus J. of Math. Phys. 48 p 043511 (preprint math-ph/0610073).

[7] Coquereaux R and Schieber G 2008 Quantum symmetries for exceptional SU(4) modular invariants associated with conformal embeddings Symmetry, Integrability and Geometry: Methods and Applications SIGMA 5 (2009), 044.

[8] Di Francesco P , Matthieu P and Senechal D 1997 Conformal Field Theory (Berlin: Springer).

[9] Di Francesco P and Zuber J -B $1990 S U(N)$-lattice integrable models associated with graphs Nucl. Phys. B 338 p 602. 
[10] Drinfeld V G 1990 On quasitriangular quasi-Hopf algebras closely related to $\operatorname{Gal}(\bar{Q} / Q)$ Algebra $i$ Analiz 2 pp 149-181.

[11] Etingof P and Ostrik V 2004 Finite tensor categories Moscow Math. J. 4 no 3 (preprint math QA/0301027).

[12] Fuchs J , Runkel I and Schweigert C 2002 TFT construction of RCFT correlators I: Partition functions, Nucl. Phys.B 646 pp 353-497 (preprint hep-th/0204148).

[13] Gannon T 1992 The classification of affine su(3) modular invariant partition functions Commun. Math. Phys. 161 pp 233-263 (preprint hep-th/9212060).

[14] Hammaoui D, Schieber and G Tahri E H 2005 Higher Coxeter graphs associated to affine $s u(3)$ modular invariants $J$. of Phys. A 38 pp 8259-8286 (preprint hepth/0412102).

[15] Isasi E and Schieber G 2007 From modular invariants to graphs: the modular splitting method J. of Phys. A 40 pp 6513-6537 (preprint math-ph/0609064).

[16] Hayashi T 1999 A canonical Tannaka duality for finite semisimple tensor categories (preprint math.QA/9904073).

[17] Hurwitz A 1886 Uber endliche Gruppen, welche in der Theorie der elliptschen Transzendenten auftreten Math. Annalen 27 pp 183-233.

[18] Kac V and Peterson D 1984 Infinite dimensional Lie algebras, theta functions, and modular forms Adv. in Math. $53 \mathrm{p} 125$.

[19] Kac V and Wakimoto M 1988 Modular and conformal invariance constraints in representation theory of affine algebras Adv. Math. $\mathbf{7 0}$ p 156.

[20] Kazhdan D and Lusztig G 1994 Tensor structures arising from affine Lie algebras, III J. Amer. Math. Soc. 7 pp 335-381.

[21] Kirillov A and Ostrik V 2002 On q-analog of McKay correspondence and ADE classification of SL2 conformal field theories Adv. in Math. Vol 171 Issue 2 pp 183-227 (preprint math.QA/0101219).

[22] Ocneanu A 1996 seminars unpublished.

[23] Ocneanu A 1999 Paths on Coxeter diagrams: from Platonic solids and singularities to minimal models and subfactors Notes taken by Goto S Fields Institute Monographs ed Rajarama Bhat et al (AMS).

[24] Ocneanu A 2000 The Classification of subgroups of quantum SU(N) Lectures at Bariloche Summer School Argentina AMS Contemporary Mathematics 294 ed Coquereaux R García A and Trinchero R.

[25] Ostrik V 2003 Module categories weak Hopf algebras and modular invariants Transform. Groups 8 no 2 pp 177-206 (preprint math QA/0111139).

[26] Petkova V B and Zuber J-B 2001 The many faces of Ocneanu cells Nucl. Phys. B 603 pp 449-496 (preprint hep-th/0101151).

[27] Schellekens A N and Warner N P 1986 Conformal subalgebras of Kac-Moody algebras Phys. Rev. D 34 no 10 pp 3092-3096.

[28] Turaev V G and Wenzl H 1993 Quantum invariants of 3-manifolds associated with classical simple Lie algebras Internat. J. Math. 4 N2 pp 323-358.

[29] Verlinde E 1988 Fusion rules and modular transformations in 2D conformal field theory Nucl. Phys. B 300360.

[30] Wolf J A 1968 The geometry and structure of isotropy irreducible homogenous spaces Acta Math. 120 pp 59-148. 1984 Erratum Acta Math. 152 pp 141-142. 